\title{
Investigation of The Numerical Solution for One Dimensional Drift-Diffusion Model in Silicon in Steady State
}

\author{
Rozana Thabet Noor ${ }^{1}$, Mumtaz M. Hussien ${ }^{2 *}$ \\ ${ }^{1,2}$ Department of Physics, College of Education for Pure Sciences, University of Mosul, Mosul, Iraq \\ E-mail: ${ }^{1}$ rozanathabet92@gmail.com, ${ }^{2 *}$ momtaz_hussien@uomosul.edu.iq
}

(Received May 06, 2020; Accepted August 31, 2020; Available online March 01, 2021)

DOI: 10.33899/edusj.2020.127055.1067, ( ) 2020, College of Education for Pure Science, University of Mosul.

This is an open access article under the CC BY 4.0 license (http://creativecommons.org/licenses/by/4.0/).

\begin{abstract}
The drift-diffusion model is considered as one of the most important models which is used to describe the characteristics of semiconductor devices and can be applied to wide range of applications started from micro up to nano scale devices after applying the suitable correction on it. The Poisson, continuity, and current equations are considered as the basic equations for semiconductor devices, these equations are partial differential equations, used in the drift diffusion model. These equations described the semiclassical electron and hole transport in semiconductor in the presence of uniformly applied electric field. In this paper a numerical method (finite difference method) has been used to find the solution of these equations depending on Gummel method and Scharfetter-Gummel scheme, the drift diffusion model is applied after many approximation and suitable boundary condition which has been considered for the $p n$ diode in both equilibrium and non-equilibrium cases at room temperature, from this simulation model a MATLAB program has been prepared to obtained diode parameters as a function of distance at the junction region, these parameters are (conduction band, carrier concentration, electric field and charge density) two diode model has been tested with different doping concentration the first with $N_{A}=N_{D}$ and the second with $N_{A}>N_{D}$ also the diode characteristic in the forward biased is obtained.
\end{abstract}

Keyword: simulation of $p n$ diode, drift diffusion model, Gummel method.

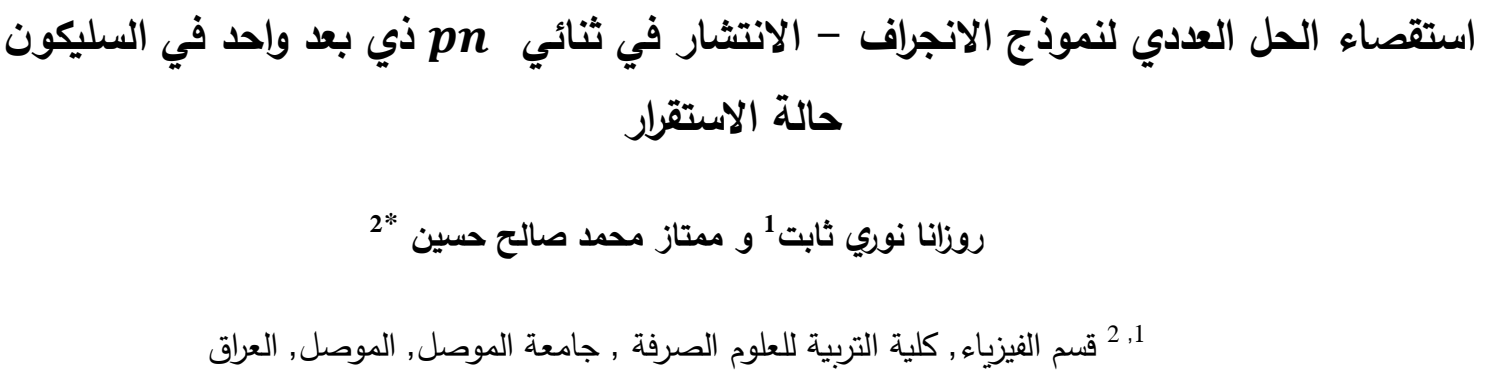

الملخص

يعد نموذج انجراف-انتشار من اهم النماذج المستخدمة لوصف خصائص نبائط اشباه الموصلات ويمكن تطبيقه على مدى واسع من التطبيقات تمتد من الابعاد الدقيقة (المايكروية) الى الابعاد النانوية بعد ادخال التصحيحات المناسبة لها. تعتبر معادلة بواسون، ومعادلة الاستمرارية ومعادلة التيار من المعادلات الاساسية في اشباه الموصلات وهي معادلات تفاضلية جزئية تستخدم في نموذج الانجراف - الانتشار. تصف هذه المعادلات الانتقال شبة الكلاسيكي للإلكترونات والفجوات في حال وجود مجال كهربائي خارجي منتظم. تم في هذا البحث تطبيق طريقة عددية (طريقة الفروق المحدودة) لإيجاد حلول لهذه المعادلات بالاعتماد على طريقة كومل 
ومخطط شيرفتر - كومل. تم تطبيق نموذج الانجراف - الانتشار ، بعد اجراء عدة تقريبات للحل وباستخدام شروط حدودية مناسبة

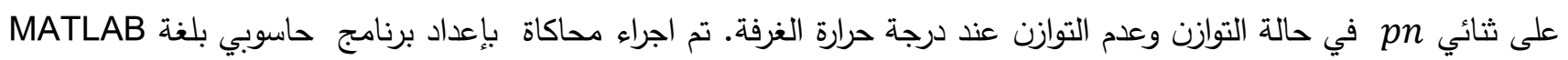
والحصول على معلمات لثنائي السليكون pn كدالة للمسافة. اذ تم الحصول على كل من حزمة التوصيل، تركيز الحاملات، المجال الكهربائي وكثافة الثحنة كدالة للمسافة في حالة التوازن اي عندما وتمت المقارنة بين الحالتين.

الكلمات الدالة: محاكاة ثنائي pn، نموذج انجر اف ـ انتشار، طريقة كومل.

المقدمة

تعد اشباه الموصلات ركيزة اساسية في التطورات التكنلوجية وثورة المعلومات ، ونتيجة للتطور الكبير والمستمر في نبائط اشباه الموصلات في جميع المجالات.. ظهرت الدراسات النظرية بموازاة الدراسات التجريبية اذ لعبت المحاكاة والطرق العددية والنمذجة ولئة ولئه الدور المهم في تطوير اداء نبائط اشباه الموصلات وذلك لتقليل الجهد والكلفة في الدراسات العملية [3-1]. وتعتمد المحاكاة في لئي دراسات اشباه الموصلات بالأساس على الاسس النظرية في فيزياء اشباه الموصلات وطبيعة حركية حاملات الثحنة في اشباه الموصلات اضافة الى شكل الحزمة لثبه الموصل ونوع وكثافة الثوائب في شبه الموصل. منذ ثلاثة عقود ومع التطور الكبير في

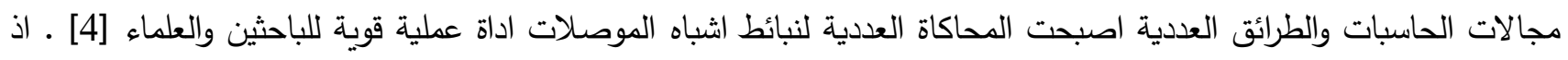
برزت تطورات من ناحية تقليص حجم النبائط الى حدود النانو مما أدخل المحاكاة في مجال ميكانيك الكم حيث ادخلت تعديلات عدئلت عديدة على النظريات السائدة بإدخال التصحيح الكمي خاصة على معادلة بولتزمان شبه الكلاسيكية والنماذج الاخرى مثل نموذج الهيدرودينميك

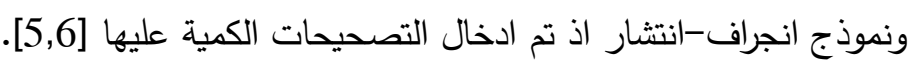

Poisson من المعادلات المهمة التي تصف نبائط اشباه الموصلات هي معادلات الاستمرارية للإلكترونات والفجوات ومعادلة بواسون

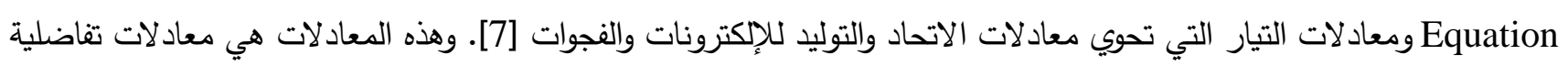

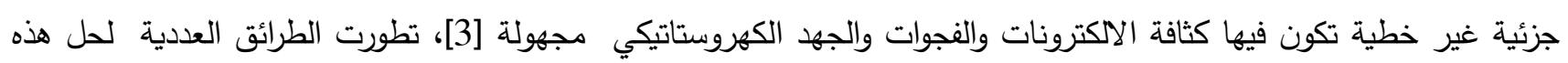
المعادلات الثلاثة نتيجة للتقدم في لغات الحاسوب والتطور الكبير بالطرائق والاساليب العددية ومن ابرز هذه الطرائق هي طريقة

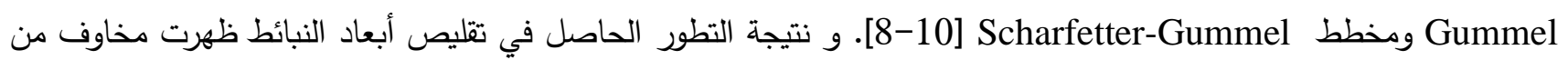
عدم صحة نموذج انجراف-انتشار مع وجود المجالات الكهربائية العالية والتي تتغير مع المكان والزمان داخل هذه النبائط اذ تم تطوير

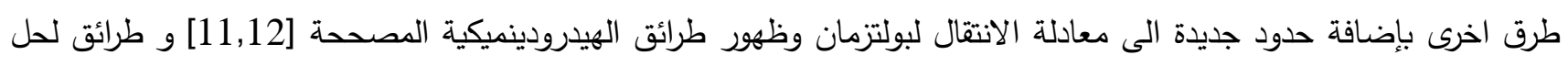

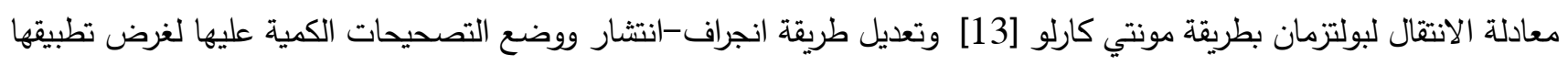
على نبائط اشباه الموصلات النانوية [14]. في هذا البحث قمنا باشتقاق معادلة بواسون ومعادلة الاستمرارية في حالتي التوازن وعدم التوازن ومن ثم تطبيق هذه المعادلات لحل

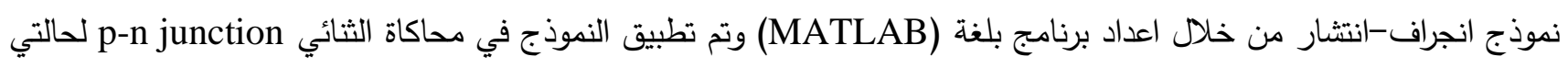
التوازن وعدم التوازن في البعد الواحد واستخدمنا مخطط شيرفتر - كومل (Scharfetter-Gummel scheme) الذي يعد مثاليا لتقدير اساسيات معادلات اشباه الموصلات لأنها تفترض ان المشتقة الجزئية للجها هي ثابتة بين النقاط المنفصلة (العقد) ولكن بالنسبة لتركيز الحاملات تم اشتقاق معادلة الاستمرارية التي توضح انها تختلف بشكل كبير بين العقد. 
يبنى نموذج الانجراف-الانتشار في اشباه الموصلات على مجموعة من المعادلات تتضمن[17-13,15] :1 - معادلة بواسون (Poisson equation)

$$
\nabla . \varepsilon \nabla V=-q\left(p-n+N_{D}^{+}-N_{A}^{-}\right)
$$

اذ تمثلع النفوذية ، V الجهـ الكهربائي، p و n كثافة الفجوات والاككترونات لوحدة الحجم،

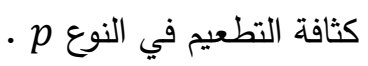
2-2 معادلة الاستمرارية (Continuity equations)

$$
\begin{aligned}
& \frac{d n}{d t}=\frac{1}{q} \nabla \cdot J_{n}+U_{n} \\
& \frac{d p}{d t}=-\frac{1}{q} \nabla \cdot J_{p}+U_{p}
\end{aligned}
$$

حيث ان $q$ شحنة الالكترون،

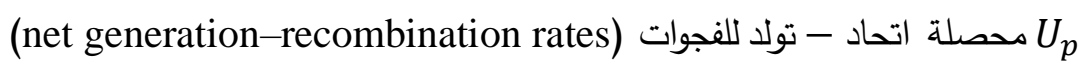
3- معادلة التيار (Current equations)

$$
\begin{aligned}
& J_{n}=q n \mu_{n} E+q D_{n} \nabla n \\
& J_{p}=q p \mu_{p} E-q D_{p} \nabla p
\end{aligned}
$$

اذ تمثل

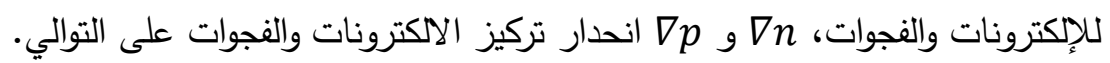
تكون معادلة التيار في نموذج الانجراف- الانتشار من حدين أساسين هما: الحد الاول هو حد الانجراف وهذا الحد ناتج عن حركة جسيمات المشحونة التانة استجابة لتطبيق المجال الكهربائي، عندما يتم تطبيق

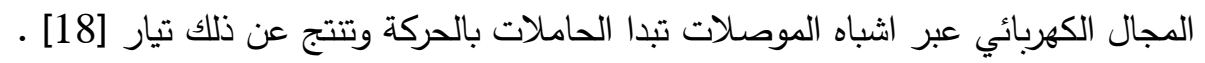

$$
J_{n}=q n \mu_{n} E \quad J_{p}=q p \mu_{p} E
$$

كثافة تيار الانجراف للإكترونات والفجوات تعتمد على اربع معلمات هي المجال الكهربائي E ، تركيز الحاملات n وp، التحركية

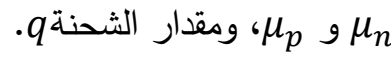

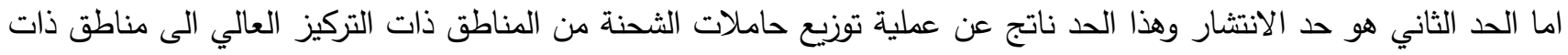

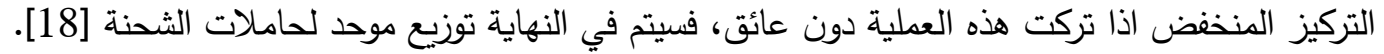
$J_{n}=q D_{n} \nabla n \quad J_{p}=-q D_{p} \nabla p$

$$
\text { نموذج الانجراف- الانتشار في البعد الواحد }
$$

يتم تطبيق نموذج الانتشار -الانجراف في بعد واحد وذلك باستخدام معادلة بواسون (1). اذ ترتبط كثافة الالكترونات n وكثافة الفجوات p، بكل من الجهد الكهروستاتيكي V، وجها شبه-فيرمي (quasi-Fermi potential) للإلكترونات 


$$
\begin{aligned}
& n=n_{i} \exp \left(\frac{q\left(\mathrm{~V}-\phi_{n}\right)}{k_{B} T}\right) \\
& p=n_{i} \exp \left(\frac{q\left(\phi_{P}-\mathrm{V}\right)}{k_{B} T}\right)
\end{aligned}
$$

اذ تمثل المعادلات (8) و(9) تركيز الاككترونات والفجوات على التوالي في حالة عدم التوازن (الحالة غير المنحلة) يمكن وضع المعادلات (8) و (9) بدلالة مستوي فرمي $E_{F}$ ومستوي فرمي عند شبه الموصل النقي $E_{\text {E }}$ وكثافة الحاملات في الحالة النقية قبل

$$
\begin{aligned}
& n=n_{i} \exp \left(\frac{E_{F}-E_{i}}{k_{B} T}\right)=n_{i} \exp \left(V / V_{T}\right) \\
& p=n_{i} \exp \left(\frac{E_{i}-E_{F}}{k_{B} T}\right)=n_{i} \exp \left(-V / V_{T}\right)
\end{aligned}
$$$$
n_{i}
$$

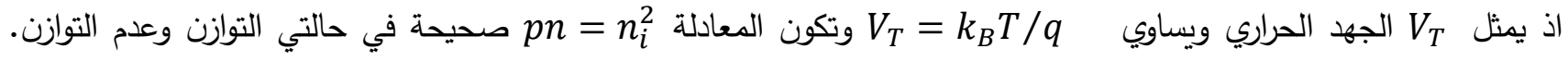

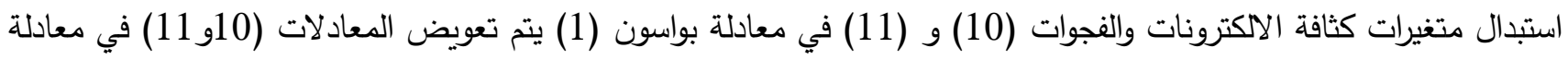

$$
\frac{d^{2} V}{d x^{2}}=-\frac{q n_{i}}{\varepsilon}\left(e^{-V / V_{T}}-e^{V / V_{T}}+\frac{C}{n_{i}}\right)
$$

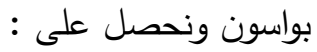

اذ تمثل

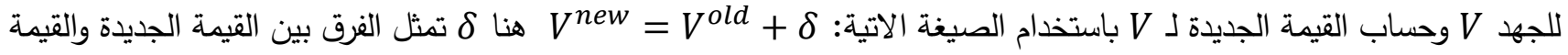
السابقة لـ V ، تصح معادلة (12) على القيمة الجديدة Vew

$$
\frac{d^{2} V^{n e w}}{d x^{2}}=\frac{V_{j+1}^{n e w}-2 V_{j}^{n e w}+V_{j-1}^{n e w}}{h^{2}}
$$

هنا ز تمثل وسم و h هي المسافة بين قيمتين متتاليتين من المسافة x ، يمكن حل معادلة بواسون بعد تعويض معادلة (13) في المعادلة (12) وترتيب الحدود ونحصل على الصيغة الاتية:

$$
\begin{gathered}
V_{j+1}^{\text {new }}-\left[2+\left(\frac{q n_{i} h^{2}}{\varepsilon V_{T}}\right)\left(e^{-V_{j}^{\text {old }} / V_{T}}+e^{V_{j}^{\text {old }} / V_{T}}\right)\right] V_{j}^{\text {new }}+V_{j-1}^{\text {new }} \\
=-\frac{q n_{i} h^{2}}{\varepsilon}\left(e^{-V_{j}^{\text {old }} / V_{T}}-e^{V_{j}^{\text {old }} / V_{T}}+\frac{C}{n_{i}}\right) \\
-\frac{q n_{i} h^{2}}{\varepsilon V_{T}}\left(e^{-V_{j}^{\text {old }} / V_{T}}+e^{V_{j}^{\text {old }} / V_{T}}\right) V_{j}^{\text {old }}
\end{gathered}
$$

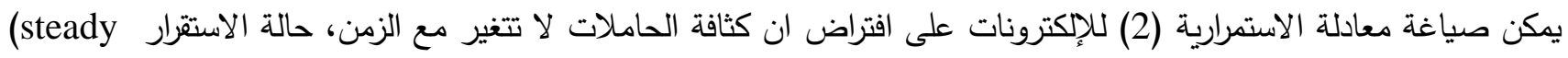
في البعد الواحد حيث 0 state)

$$
\begin{aligned}
& \frac{d J_{n_{x}}}{d x}=-q U_{n} \\
& \frac{J_{i+\frac{1}{2}}^{n}-J_{i-\frac{1}{2}}^{n}}{h}=-q U_{n}
\end{aligned}
$$

يتم استخدم طريقة الفروق المحدة لتمثيل المعادلة (15) لنحصل على الصيقة الاتية

يشير الترميز العلوي للدالة على النوع n او p اما الترميز السفلي للدالة فيشير الى العقد. يعتبر مخطط شيرفتر - كومل (Scharfetter- Gummel) 


$$
\begin{aligned}
& J_{i-\frac{1}{2}}^{n}=\frac{q D_{i-\frac{1}{2}}^{n}}{h}\left[n_{i} B\left(\frac{V_{i}-V_{i-1}}{V_{T}}\right)-n_{i-1} B\left(\frac{V_{i-1}-V_{i}}{V_{T}}\right)\right] \\
& J_{i+\frac{1}{2}}^{n}=\frac{-q D_{i+\frac{1}{2}}^{n}}{h}\left[n_{i+1} B\left(\frac{V_{i+1}-V_{i}}{V_{T}}\right)+n_{i} B\left(\frac{V_{i}-V_{i+1}}{V_{T}}\right)\right] \\
& \text { بتعويض قيم التيارات في معادلة الفروق (16) نحصل على معادلة الاستمرارية للإلكترونات. } \\
& \frac{q D_{i-\frac{1}{2}}^{n}}{h} B\left(\frac{V_{i-1}-V_{i}}{V_{T}}\right) n_{i-1}-\left[\frac{q D_{i+\frac{1}{2}}^{n}}{h} B\left(\frac{V_{i}-V_{i+1}}{V_{T}}\right)+\frac{q D_{i-\frac{1}{2}}^{n}}{h} B\left(\frac{V_{i}-V_{i-1}}{V_{T}}\right)\right] n_{i} \\
& +\frac{q D_{i+\frac{1}{2}}^{n}}{h} B\left(\frac{V_{i+1}-V_{i}}{V_{T}}\right) n_{i+1}=U_{i} \\
& \text { وبنفس الطريقة يمكن الحصول على معادلة الاستمرارية للفجوات كما مبين في المعادلة الاتية: } \\
& \frac{q D_{i-\frac{1}{2}}^{p}}{h^{2}} B\left(\frac{V_{i}-V_{i-1}}{V_{T}}\right) p_{i-1}-\left[\frac{q D_{i+\frac{1}{2}}^{p}}{h^{2}} B\left(\frac{V_{i+1}-V_{i}}{V_{T}}\right)+\frac{q D_{i-\frac{1}{2}}^{p}}{h^{2}} B\left(\frac{V_{i-1}-V_{i}}{V_{T}}\right)\right] p_{i} \\
& +\frac{q D_{i+\frac{1}{2}}^{p}}{h^{2}} B\left(\frac{V_{i}-V_{i+1}}{V_{T}}\right) p_{i+1}=U_{i}
\end{aligned}
$$

اذ تمثل Bernoulli function$$
B(x)=\frac{x}{e^{x}-1}
$$

$$
\text { pn تطبيق نموذج الانجراف- الانتشار على الثنائي }
$$

في هذا البحث تم تطبيق نموذج الانجراف-الانتشار على الثنائي pn في حالة الاستقرار وفي حالتي التوازن وعدم التوازن. في حالة التوازن (equilibrium case) أي في حالة غياب الجهد الخارجي المسلط عند درجة حرارة معينة تكون محصلة تيار المفرق صفرا اي يلغي تيار الانجراف الناتج من المجال الكهبائي للحاجز تيار الانتشار الناتج من انحدار تركيز الحاملات لكلا النوعين من

$$
J=J_{e}+J_{h}=0
$$

لوصف خصائص الثنائي nn يجب معرفة عرض منطقة الاستنزاف وتوزيع الثحنة للإلكترونات والفجوات والمجال الكهربائي. عندما

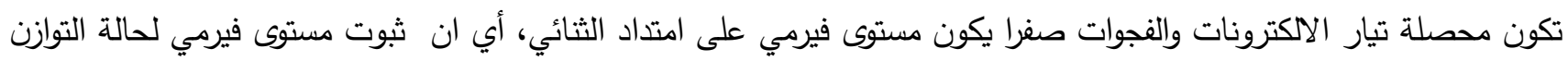

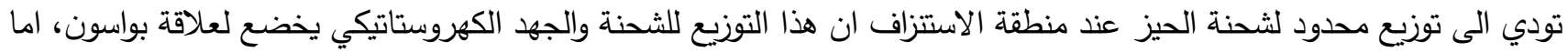
في المناطق خارج منطقة الاستزاف فان كثافة الثحنة تساوي صفر أي ان الثحنات تبقى متعادلة. هبوط الجهد خلال منطقة الاستزاف

$$
V_{b i}=\frac{k T}{q} \ln \frac{N_{A} N_{D}}{n_{i}^{2}}
$$
في حالة التوازن يعرف بـ جهد الحاجز والذي يعطى بالعلاقة الاتية:

اذ تمثل V ل جهد الحاجز او الجهد الداخلي (build-in potential)، وان جهد الحاجز سيأخذ دائما الوضع او القيمة الذي يكفل التعادل بين تياري التوصيل والانتشار عند عدم وجود فولتية مسلطة، حيث ان زيادة تيار الانتثار بسبب زيادة درجة الحرارة او الثوائب يؤدي الى زيادة عدد الايونات الموجبة والسالبة على طرفي منطقة الاستنزاف وبالتالي الى زيادة جهد الحاجز ويودي بالمقابل الى زيادة تيار التوصيل بالاتجاه المعاكس تركيز الحاملات في حالة التوازن معادلة (10) و (11). 


$$
n=n_{i} \exp \left(\frac{q \mathrm{~V}}{k_{B} T}\right) \quad p=n_{i} \exp \left(-\frac{q \mathrm{~V}}{k_{B} T}\right)
$$

بعد التعويض عن قيم n و p المعادلة (24) وتبسيط المعادلة مرورا بالمعادلة (14) نحصل على صيغة بسيطة تثبه المعادلة (25) تكون بدلالة الجهد، باستبدال هذه القيم في معادلة بواسون الخطية وحل هذه المعادلة بطريقة تكرارية وحسب الثروط الحدودية للجهد

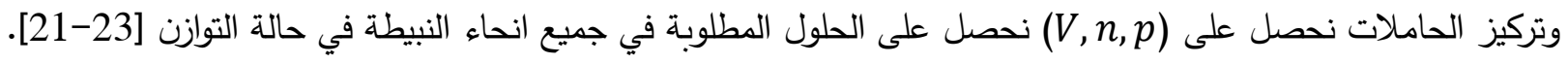
اما في حالة عدم التوازن (non-equilibrium case) فان وجود طبقة الاستنزاف يرافقه نشوء جهد حاجز يعيق انتشار الحاملات

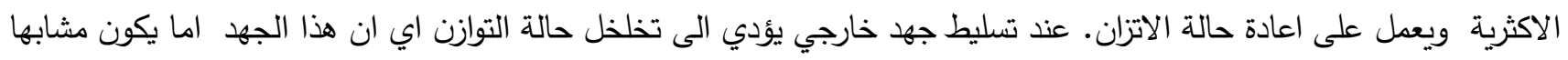

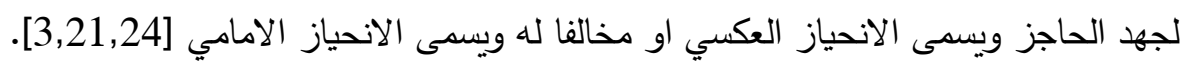

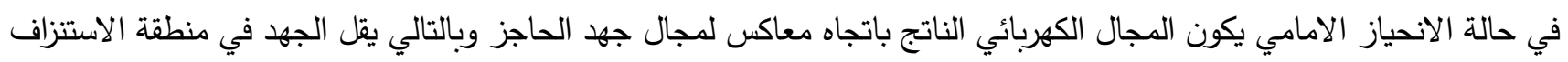
اي $V_{b i} V_{F}$

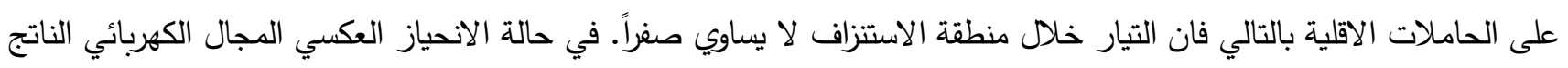

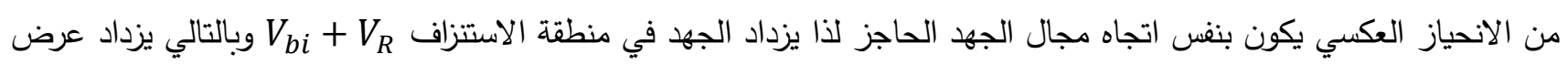
منطقة الاستنزاف [21].

الخوارزمية المستخدمة لحل نموذج الانجراف الانتشار في حالة عدم التوازن

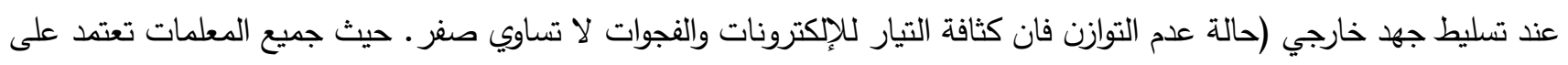

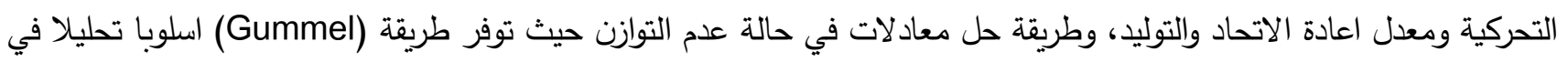
تحويل المعادلات غير الخطية الى معادلات خطية وكما يلي: ولدئ اولا / نحل معادلة بواسون غير الخطية. ثانيا / يتم استبدال الجهد الذي تم الحصول عليه من حل معادلة بواسون في معادلة الاستمرارية والتي اصبحت الان خطية باجراء التقرببات المناسبة باعتبار $\delta$ صغيرة جدا، ويتم حلها مباشرة باستخدام الطريقة التكرارية. ثالثا / نتائج الجهد التي تم الحصول عليها تعتبر قيم اولية مرة اخرى لايجاد القيم الجديدة في معادلة بواسون ويتم عن طريق تكرار العملية الوصول الى الحل النهائي.

نظرا لان هذه المسالة في بعد واحد يتم استخدام تحليل lower and upper decomposition method (LU) لانه تم تطبيق نموذج الانجراف-الانتشار في بعد واحد.

$$
A \phi=f
$$

يمكن تثكيل المصفوفة A كحاصل ضرب المصفوفتين LU ، حيث U مصفوفة مثلثية علوية و L التمفوفة مثلثية سفلية على

$$
\text { التوالي كما ان المصفوفة A هي بالاساس مصفوفة قطرية ثلاثية ويمكن تمثيلها كما يلي [3] : }
$$

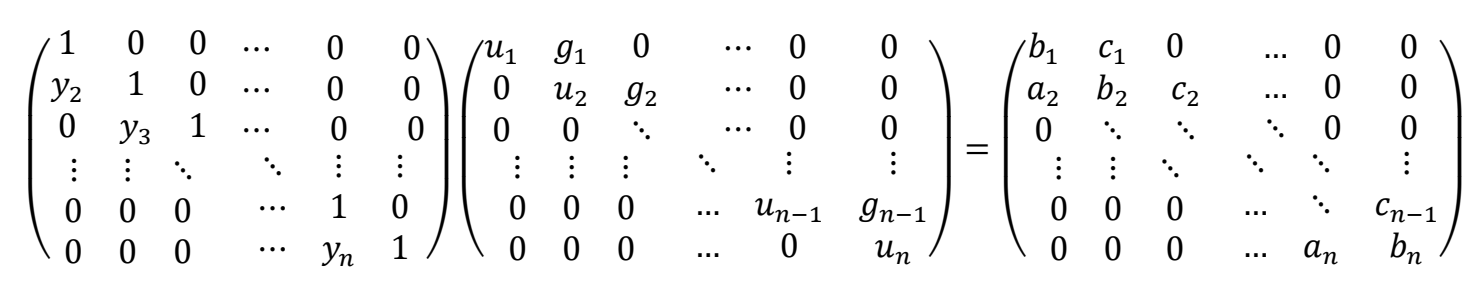


يمكن بأجراء الضرب المباشر لإيجاد قيم عناصر المصفوفتين L L Uدلالة عناصر المصفوفة A حيث يمكن ايجاد عناصر

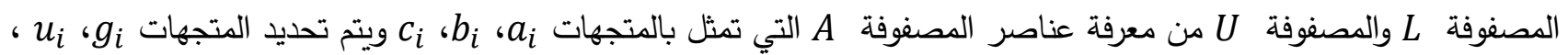

$$
\begin{gathered}
g_{i}=c_{i}, i=1,2, \ldots, n \\
u_{1}=b_{1} \quad, \quad y_{2}=\frac{a_{2}}{u_{1}} \\
u_{i}=b_{i}-\frac{a_{i}}{u_{i-1}} c_{i}, \quad y_{i+1}=\frac{a_{i+1}}{u_{i}}, \quad i=2,3, \ldots, n
\end{gathered}
$$

من خلا ذلك يمكن حل معادلة بواسون بطريقة المصفوفات وبطريقة تكرارية للحصول على حل بأفضل تقارب، وكذلك حل مسائل الاستمرارية للإكترونات والفجوات [3] ويبين الثكل (1) مخطط خوارزمية الحل حيث يتم تحديد خطوة المسافة التي تحدد لتكون اقل من طول ديباي وهذا الثرط مهم للحصول على التقارب المطلوب في الحل بالطريقة التكرارية، اما كثافة التطعيم في جهتي الثنائي تحدد بتحديد تركيز التشويب م

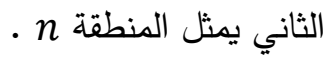

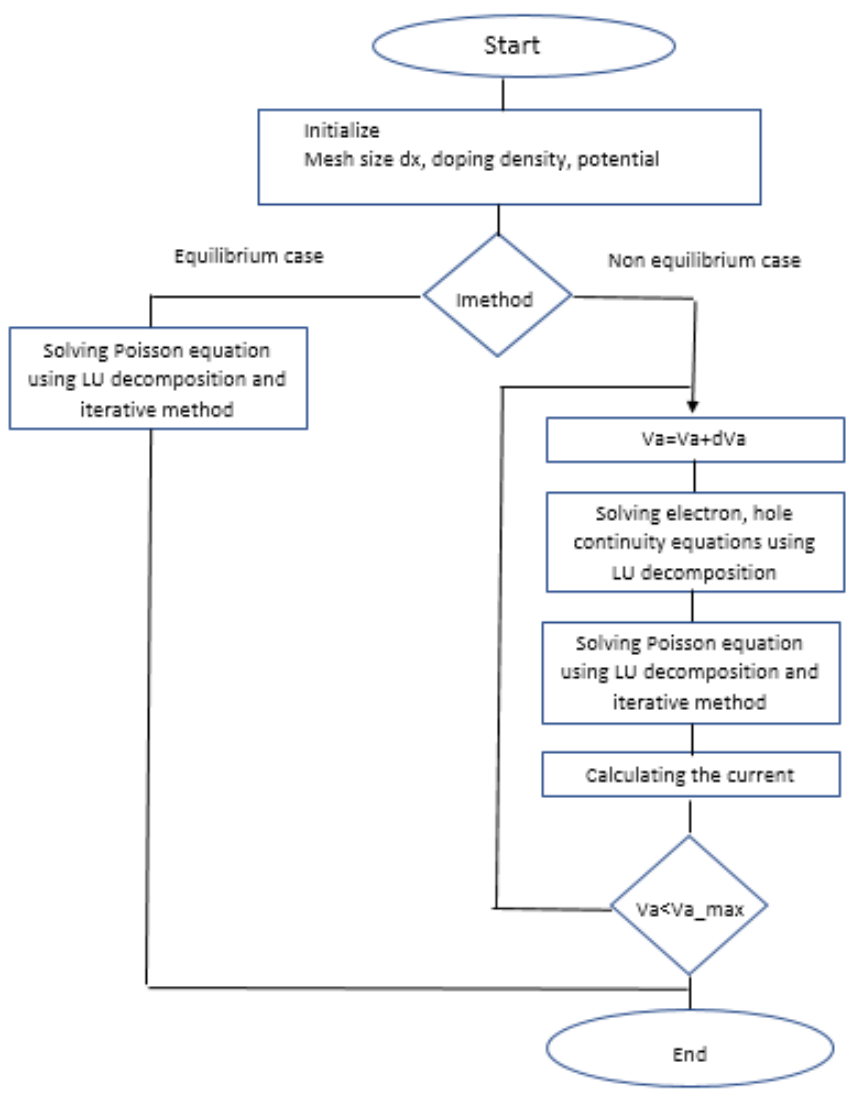

الثكل (1) مخطط خوارزمية الحل

النتائج والمناقشة

تم الحصول على العديد من المعلمات (مثل حزمة التوصيل، توزيع الثحنة، شدة المجال الكهربائي و كثافة الثحنة كدالة للمسافة) وذلك باستخدام المحاكاة لنموذج الانجراف-الانتشار . اذ تم اقتراح نموذجين من ثنائيات السليكون نوع pn وكما موضح في في الجدول (1). تم دراسة حالتين تعتمد على نسبة التشويب لكل من القابلات والمانحات N N N على التوالي وهذه الحالات هي عندما تكون 
نسبة التشويب متساوية أي برنامج بلغة وتشبيق نموذج المحاكاة على هاتين الحالتين عند درجة حرارة الغرفة في حالتي التوازن وعدم التوازن.

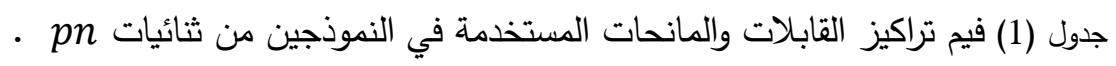

\begin{tabular}{|c|c|c|}
\hline Diode No. & $\mathrm{N}_{\mathrm{A}}\left(\mathrm{cm}^{-3}\right)$ & $\mathrm{N}_{\mathrm{D}}\left(\mathrm{cm}^{-3}\right)$ \\
\hline 1 & $10^{16}$ & $10^{16}$ \\
\hline 2 & $1 \times 10^{17}$ & $10^{16}$ \\
\hline
\end{tabular}

في تم حساب حزمة التوصيل كدالة للمسافة للنموذجين قيد الدراسة ابتدآ من المنطقة p وباتجاه المنطقة n في حالة عدم وجود فولتية خارجية مسلطة على ثنائي مn. الثكل (2) ان منطقة الاستتزاف تكون صغيرة جدا، كما ان ارتفاع حزمة التوصيل من المنطقة

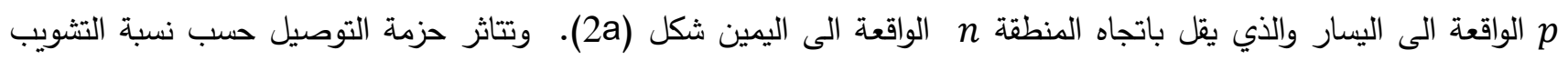
فعندما تكون $N_{A}>N_{D}$ فهنالك ارتفاع في حزمة التوصيل من جهة المنطقة $p$ م ، ويزداد هذا الارتفاع مع زيادة نسبة التشويب

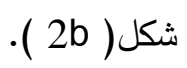
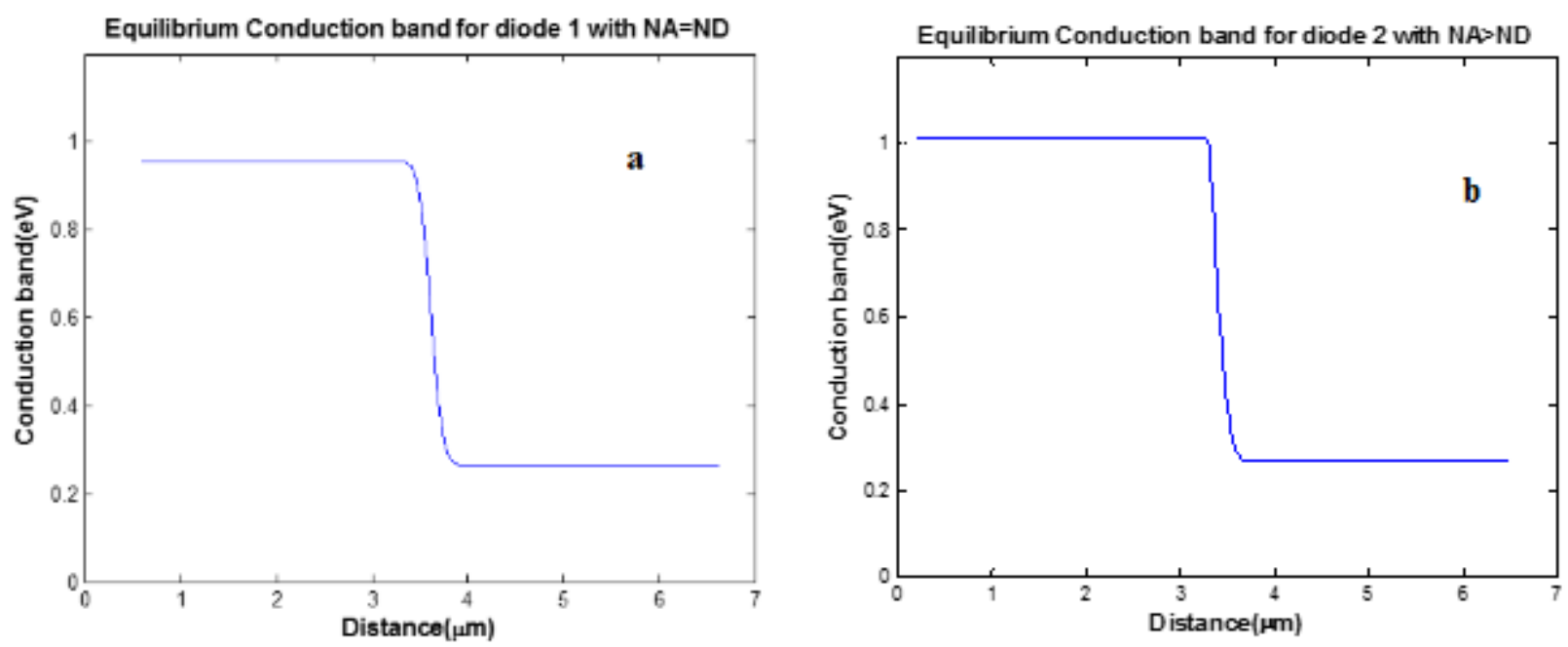

$$
\text { الشكل (2) محاكاة حزمة التوصيل كدالة المسافة للثنائيات عندما (a) }
$$

اما في المنطقة n فان القيمة تبقى كما هي وذلك لان التشويب في هذه المنطقة هو نفسه للنموذجين في هذه الدراسة. في حالة زيادة فان هذا المستوي في المنطقة n ينخفض الى الاسفل ليعطي حاجزا اعلى في منطقة المفرق. يبين الثكل (3) توزيع الثحنة حول منطقة الاستتزاف في حالة عدم وجود فولتية خارجية مسلطة على الثنائي. ينشا نتيجة انتقال الالكترونات والفجوات عبر منطقة

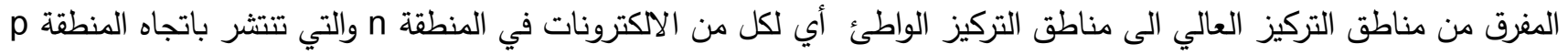
والفجوات تتنقل من المنطقة p الى المنطقة n نتيجة لعملية الانتشار . وهذا يؤدي الى نشوء جهد المفرق، اذ تتجمع شحنات عن شحنات سالبة في المنطقة p وشحنات موجبة في المنطقة n ـ يبين الثكل (3) توزيع الثحنات كدالة للمسافة على طرفي المفرق وفي منطقة الاستتزاف والتي يطلق عليها ايضا بمنطقة فضاء الثحنة space charge region ، اذ يظهر في منطقة الاستنزاف توزيع للثحنات السالبة في المنطقة p بالمقابل هنالك توزيع للشحنات الموجبة في المنطقة n م اما خارج منطقة الاستنزاف فان توزيع الثحنات يساوي صفرا الثكل (3a). كما يمكن ملاحظة ان توزيع الثحنة يكون حالة الثنائي الاول عندما تكون $N_{A}=N_{\text { }}$ يكون 
التوزيع متماثل وبإثارة مختلفة عندما تتناسب مع زيادة التشويب، الثكل (3b) مما يعطي توزيع غير متماثل للشحنات في منطقة المفرق. ينتج عن تراكم الثحنات في

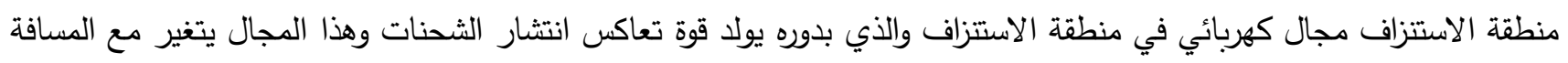

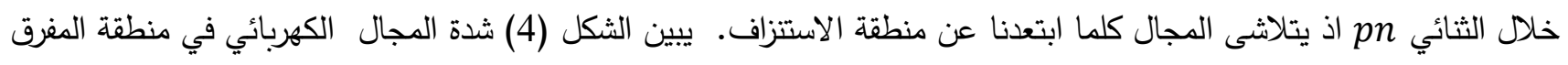
كدالة للمسافة للثنائيات المستخدمة في الدراسة، اذ يمكن ملاحظة (بغض النظر عن الاشارة السالبة لقيمة المجالالكهربائي والتي تثير

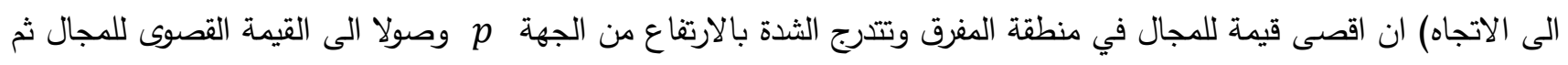
تتدرج بالانفاض في الجهة n وصولا الى الصفر ويكون هذا السلوك متجانساً في حالة الثنائي الاول حيث ، وغير متجانس في حالة الثنائي الثاني حيث عدم وجود فولتية خارجية، في حين تتزداد قيمة ذروة المجال بالاتجاه السالب بثكل حاد مع زيادة نسبة التشويب في منطقة المفرق، الثكل (4b). من المعلمات الاخرى التي يمكن حسابه هي كثافة توزيع الحاملات على طرفي المفرق، اذ يبين الثكل (5) شكل التوزيع لكل من الفجوات والاكترونات في الثنائي كدالة للمسافة عبر منطقة المفرق ويمكن ملاحظة ان تركيز الفجوات في المنطقة p تاخذ قيمة الاكثرية في هذه المنطقة وتهبط هذه القيمة الى القيمة الدنيا تركيز الاقلية في المنطقة n ميبين الثكل (5) شكل التغير

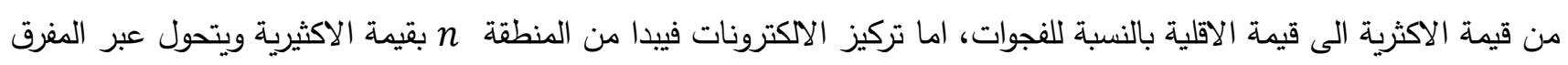
الى تركيز الاقلية في منطقة p ، ويمكن ملاحظة هبوط تركيز الحاملات سواءا الالكترونات او الفجوات الى القيمة الدنيا في منطقة

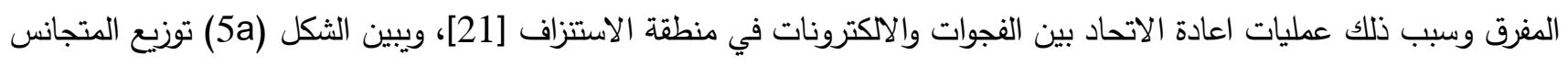

عبر المفرق في حالة الثنائي الاول، الثكل (5a) في حين يكون التوزيع غير المتجانس في حالة الثنائي الثاني، الثكل (5b).

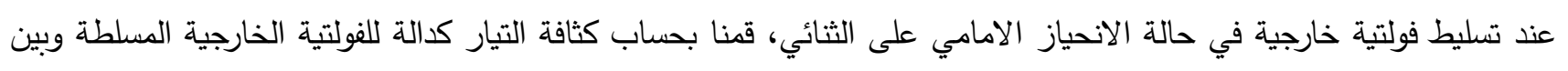

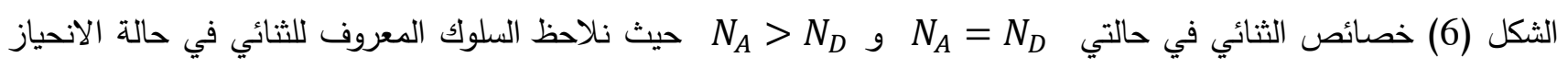
الامامي وهذه الحسابات تمت عن طريق حل نموذج الانتشار -الانجراف وبدون استخدام معادلة تجريبية حيث تعتمد هذه المعادلة على في اختيار منحني مناسب بطريقة المربعات الصغرى غير الخطية لتمثيل سلوك الثنائي.
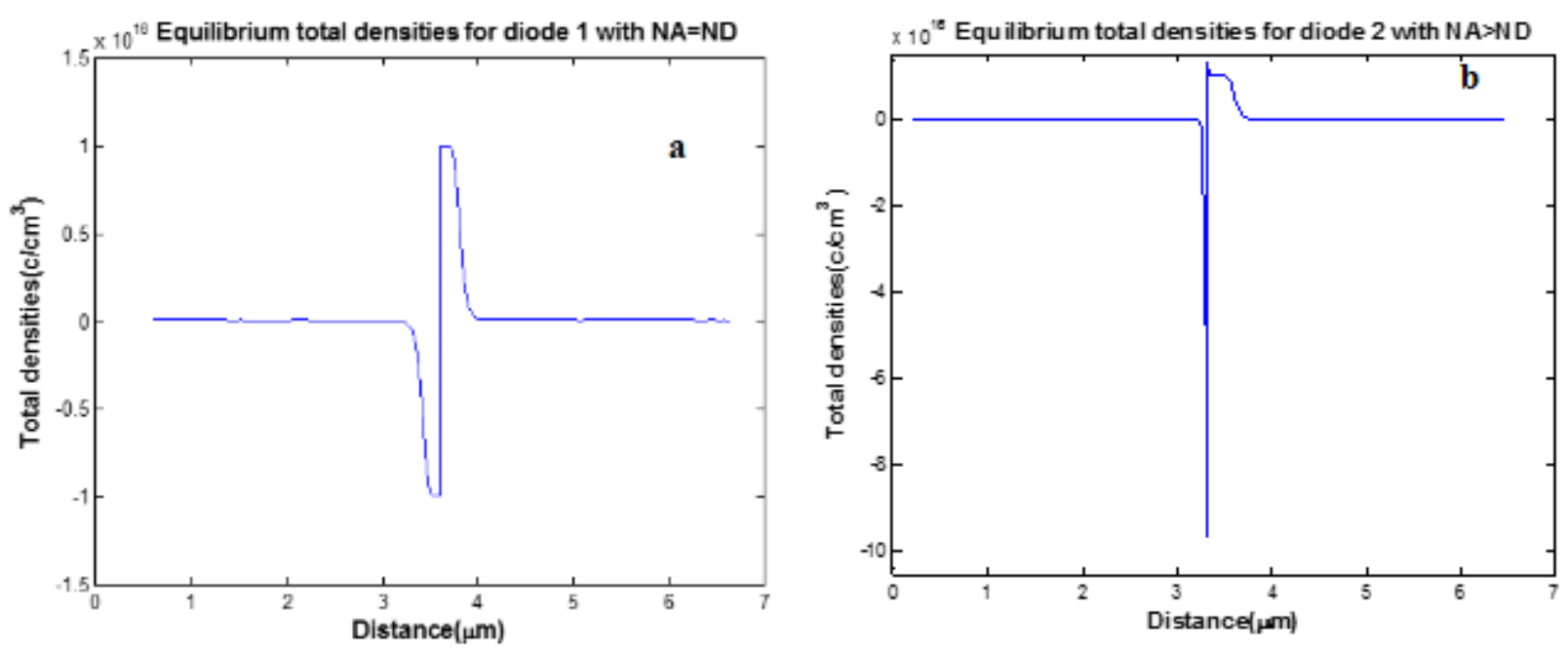

الثكل (3) توزيع الثحنة مع للثنائي pn عندما (a) 

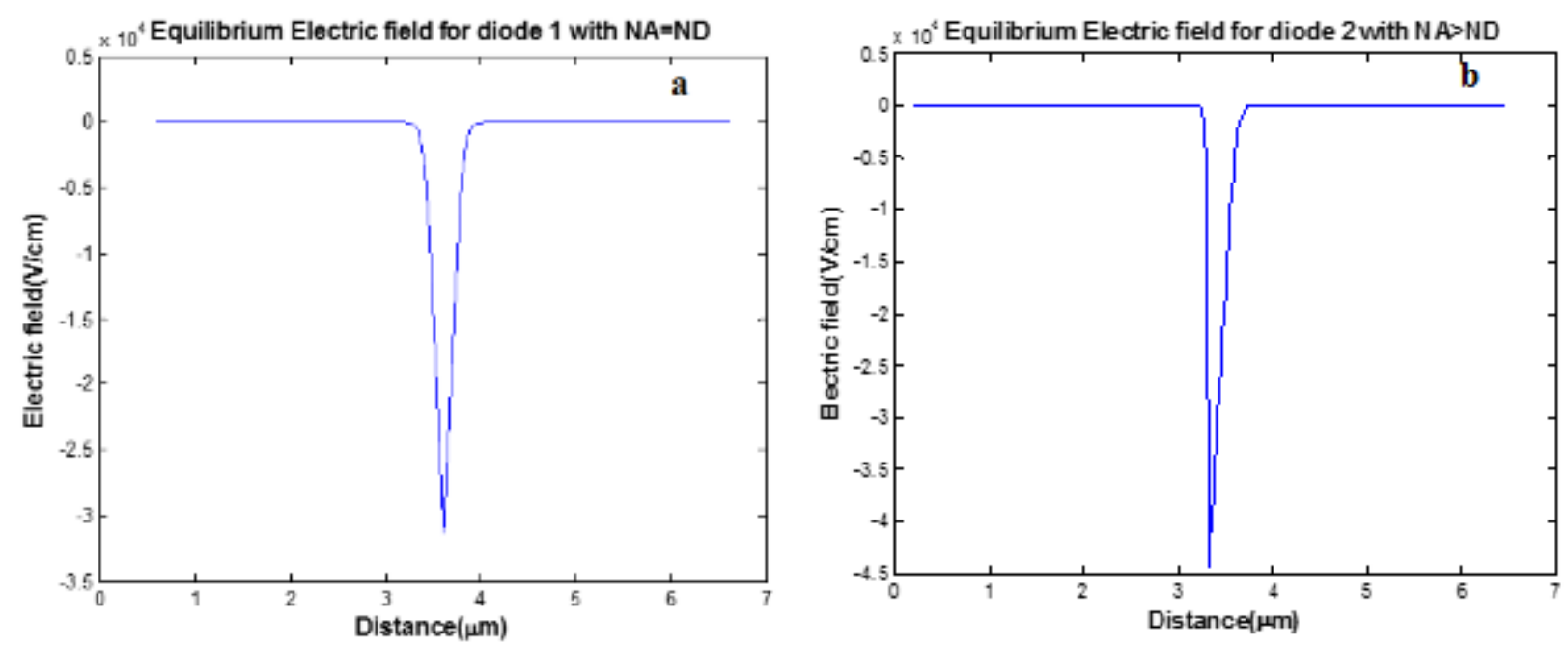

الشكل (4) توزيع شدة المجال الكهربائي في منطقة الاستنزاف للثنائي pn كدالة للمسافة عندما (a)
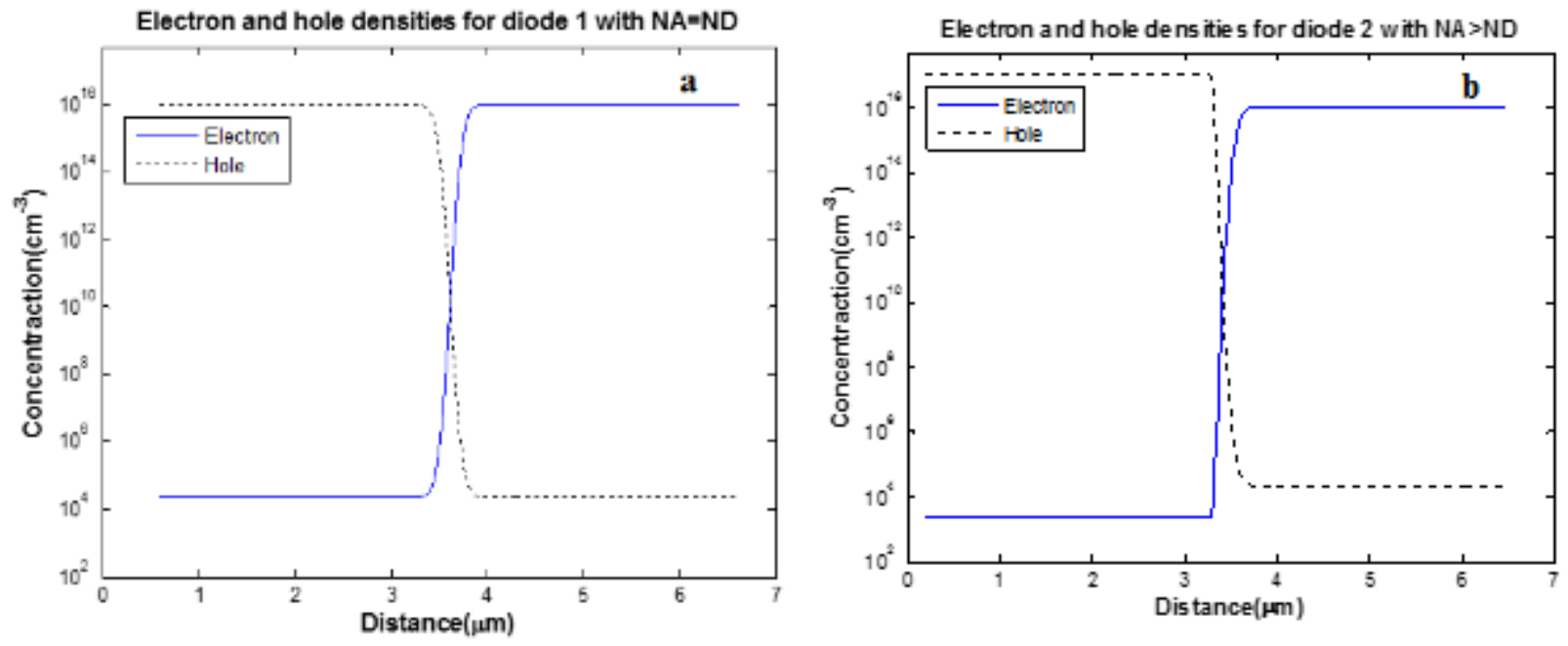
$. N_{A}>N_{D}$
(b) ${ }^{\prime} N_{A}=N_{D}$
(a) توزيع كثافة الشحنة في منطقة المفرق كدالة للمسافة

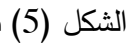
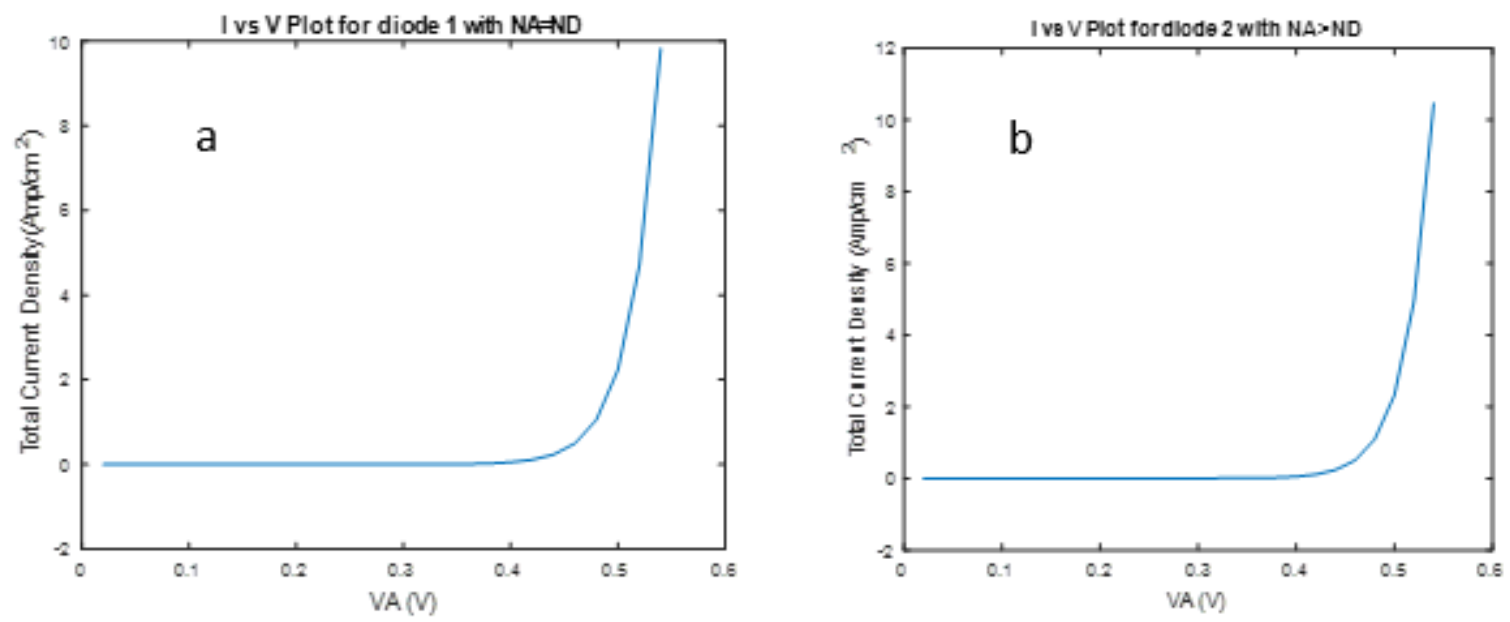

الثكل (6) كثافة التيار كدالة للفولتية المسلطة في حالة الانحياز الامامي للثنائيات (a) الثنائي 
تم الحصول على محاكاة لنماذج من الثنائيات pn للسليكون في حالة التوازن وعدم التوازن تم التوصل الى معلمات الثنائيات والعلاقة بين كثافة التيار والفولطية المسلطة في حالة الانحياز الامامي وذلك باستخدام نموذج انجراف- انتشار وحسب طريقة (Gummel)

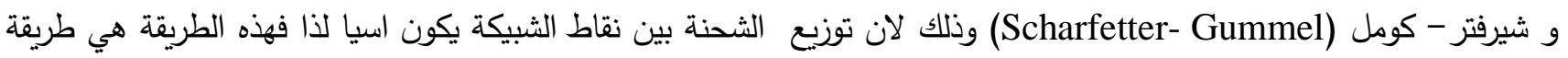
مثالية لحل مثل هذا السلوك الاسي اما الجهد المسلط فيمكن تقريبه من خلال السلوك الخطي مع مجال ثابت والنتائج التي تم الحصول

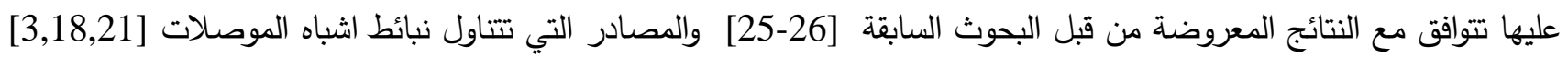
اذ تبين أن نتائج نموذج انجراف- انتشار ذي البعد الواحد يمكن ان تعطي نتائج مقاربة للنتائج العملية والحسابات النظرية في الابعاد الثلاثة.

يتقدم الباحثان بالثكر والتقدير لعمادة كلية التربية للعلوم الصرفة وقسم الفيزياء على دعم البحث وكذلك جزيل الثكر والعرفان للدكتور على عباس من قسم الفيزياء كلية التربية للعلوم الصرفة جامعة الموصل للملاحظات القيمة التي اغنت البحث.

\section{المصادر}

1- Shapo, B., C. Ball, I. Kizilyalli, and Umberto Ravaioli, Superlattices and Microstructures 4, no. 1: 39-43 (1988).

2- Akura, Mise, Geoffrey Dunn, Mohammed Missous, and J. Sexton, IEEE Electron Device Letters 38, no. 4: 438-440 (2017).

3- Vasileska, Dragica, Stephen M. Goodnick, and Gerhard Klimeck. Computational Electronics: semiclassical and quantum device modeling and simulation. CRC press, 2016.

4- Shakouri, Ali. Annual review of materials research 41 (2011).

5- Odanaka, Shinji. IEEE Transactions on Computer-Aided Design of Integrated Circuits and Systems 23, no. 6: 837-842 (2004).

6- de Falco, Carlo, Joseph W. Jerome, and Riccardo Sacco. Journal of Computational Physics 228, no. 5: 1770-1789 (2009).

7- Lundstrom, Mark. In 2015 International Conference on Simulation of Semiconductor Processes and Devices (SISPAD), pp. 1-3. IEEE, (2015).

8- Miller, J. J. H., and Song Wang. ESAIM: Mathematical Modelling and Numerical Analysis 28, no. 2: 123-140 (1994).

9- Koprucki, Thomas, Nella Rotundo, Patricio Farrell, Duy Hai Doan, and Jürgen Fuhrmann. Optical and Quantum Electronics 47, no. 6: 1327-1332 (2015).

10- Gummel, Hermann K., IEEE Transactions on electron devices 11, no. 10: 455-465 (1964).

11- Gyi, Myo Thein, and Ansgar Jüngel., Advances in Differential Equations 5, no. 4-6: 773-800 (2000).

12- Zhang, Bo, and Joseph W. Jerome., Nonlinear analysis 26, no. 4: 845-856 (1996). 
13- Moglestue, C. Monte Carlo simulation of semiconductor devices. Springer Science \& Business Media, (2013).

14- Chen, Ren-Chuen, and Jinn-Liang Liu Journal of Computational Physics 204, no. 1: 131-156 (2005).

15- Peter Fischer, Z. I. T. I. "Numerical solution of space charge density and field in arbitrarily doped diode structures." (2014).

16- Noda, Yoshikatsu. "On iterative schemes for a stationary problem to a quantum drift diffusion model." Journal of Computational Electronics 11, no. 4: 385-396 (2012).

17- Dalle, C., and P. A. Rolland., International Journal of Numerical Modelling: Electronic Networks, Devices and Fields 2, no. 2: 61-73 (1989).

18- Neamen, Donald A. Semiconductor physics and devices: basic principles. New York, NY: McGraw-Hill, (2012).

19- Selberherr, Siegfried. Analysis and simulation of semiconductor devices. Springer Science \& Business Media, (2012).

20- Hulbert, Robert. "Designing a Simulator for an Electrically-Pumped Organic Laser Diode." (2019).

21- Sze, Simon M., and Kwok K. Ng. Physics of semiconductor devices. John Wiley \& sons, (2006).

22- Van Zeghbroeck, Bart. "Principles of electronic devices." University of Colorado (2011).

23- Pierret, Robert F., and Gerold W. Neudeck. Advanced semiconductor fundamentals. Vol. 6. Reading, MA: Addison-Wesley, (1987).

24- Farrell, Patricio, Nella Rotundo, Duy Hai Doan, Markus Kantner, Jürgen Fuhrmann, and Thomas Koprucki. "Numerical methods for drift-diffusion models." (2016).

25- Khorasani, Sina, Solid-State Electronics 122: 37-44, (2016).

26- Mazhari, Baquer, and Aditya Mahajan, IEEE Transactions on Education 48, no. 1: 60-62, (2005). 